\title{
Norfloxacin in Multiple Drug Resistant Typhoid Fever
}

\author{
M.A. Kamili, M.D., M.Y. Shah, M.D., Gazanfar All, M.D., R.L. Koul, M.D., Aejaz Aziz, M.D. \\ G.Q. Allaqaband, F.R.C.P., Altaf Hussain, M.B.B.S. \\ Kashmir, India
}

DOI: http://dx.doi.org/10.5915/23-1-14378

\begin{abstract}
This study was undertaken to assess the efficacy of Norfloxacin, and oral quinolone, in multiple drug resistant salmonella typhi infections in 45 patients. All these patients received $800 \mathrm{mg}$ of Norfloxacin daily for a period of two weeks. All patients had positive blood cultures for salmonella typhi and 30 patients had, in addition, high and rising titers of $T_{O}$ and $T_{H}$ antigen $(>1: 160)$.

All patients responded from day 4 to day 10 of Norfloxacin therapy. A follow-up of one year did not reveal any relapse or a carrier state in the studied group. We recommend use of Norfloxacin in chloramphenicol resistant salmonella typhi infections.
\end{abstract}

Key words: Salmonella typhi, Norfloxacin, multiple drug resistance.

Norfloxacin is an oral fluro-quinolone carboxylic acid compound with a wide bacterial spectrum against both gram positive and gram negative organisms. ${ }^{1-4}$

Antibiotic resistance to Norfloxacin is uncommon because of its inhibitory action on bacterial DNA gyrase, ${ }^{3-7}$ an enzyme essential for bacterial for DNA replication.

Salmonella typhi infections are important problems in clinical medicine, and the introduction of new antimicrobials has resulted in the emergence of resistance to the antibiotics, which is often mediated by R-plasmids.",' The resistance to chloramphenicol is reported in the U.S. ${ }^{10}$ and in India. It has been reported at an alarming rate of $30 \% .^{11}$

Published data are insufficient to assess the efficacy of Norfloxacin for therapy of typhoid fever and typhoid carriers. ${ }^{12}$ Our study discusses the role of

From Government Medical College and S.M.H.S. Hospital, Srinagar

Kashmir, India

Reprint Requests: Dr. M.A. Kamili

51-Balgarden, Srinagar

Kashmir-190 010, India
Norfloxacin in multiple drug resistant salmonella typhi infections in $\mathbf{4 5}$ patients.

\section{Methods}

This study was conducted in the Department of Medicine, SMHS Hospitals, Srinagar. Forty-five patients clinically diagnosed with enteric fever (prolonged fever, GI symptoms, prostration, and spleenomegaly), and confirmed by bacteriological/serological tests, were enrolled in the study. All had been treated for enteric fever with chloramphenical/contrimaxazol with furoxone in adequate doses for a period of at least eight days before reporting to this hospital. None of these patients had shown a clinical response and were persistently febrile and toxic. Culture negative patients were not included in the study.

\section{Microbiological methods}

For blood culture with all sterile precautions, $10 \mathrm{ml}$ of a patient's blood was taken, $5 \mathrm{ml}$ each introduced in Bile Broth Media and Glucose Broth Media. It was incubated at $37^{\circ} \mathrm{C}$, making daily subcultures onto MacConkey's and blood Agar Media. Non-lactose fermenting colonies were studied in detail for morphology, motility, biochemical reactions, and seroaggulutination with specific antisera. Antibiotic sen- 
sitivity was done by disc-diffusion method (Kirby and Bauer Methods). All isolates were resistant to Ampicillin, Chloramphenicol, Cotrimoxazol, Tetracycline, and Streptomycin. However, bacteria were sensitive to Norfloxacin (10 microgam disc). Phage typing of Salmonella typhi strains was done at National Salmonella Phage Typing Centre, New Delhi (India), which also confirmed the identification of strains and drug resistance to Ampicillin, Chloramphenical, Tetracylcine, and Streptomycin. All isolated strains were identified to be of Biotype II Phage Type Untypeable (UVS ${ }_{2}$ ).

For urine cultures, urine was collected under aspetic precaution and the centrifuged deposit was innoculated onto Blood Agar and MacConkey's Media. The rest of the procedure was the same as that of the blood culture. One to two gram stools were innoc'?ated at bedside into G.N. Broth. After incubating at $37^{\circ} \mathrm{C}$ it was subsequently subcultured onto Blood Agar and MacConkey's Media for 24 hours and $\mathbf{4 8}$ hours. Subsequent procedures were the same as those for blood.

Nine patients in the study group presented with or developed neurological complications in the form of Parkinsonism (2), myelitis (1), meningoencephalitis (2), cerebellitis (2), psychosis (1), and polymyositis (1). Because of the non-availability of either newer cephalosporins or other quinolone derivatives, Norfloxacin was given orally in doses of $400 \mathrm{mg}$ every 12 hours for a period of two weeks. To monitor compliance and to check for possible reactions to the drug, patients were admitted to the hospital. Urine analysis, complete blood counts, liver function tests, and tests for glucose, blood urea nitrogen, creatinine and uric acid were done for all patients before treatment and at days 7 and 14 of therapy.

Patients were followed at one year to detect a carrier state. Stool and urine cultures were carried out during this follow-up study.

\section{Results}

After four days of Norfloxacin treatment all patients had a sense of well-being. Twenty patients $(45 \%)$ became afebrile on day 4 and 25 patients $(55 \%)$ became afebrile by day 7 . The drug was continued for two weeks in all patients. The nine patients with neurological complications had a total recovery within another two weeks after stopping Norfloxacin. Patients with septecemia also recovered in the same period. No side effects from Norfloxacin therapy were encountered.

At the time of the 12 month follow-up, no patient had experienced a relapse. No carrier state was detected by culture. There also was no treatment failure nor any mortality.

\section{Discussion}

Salmonella typhi infections remain one of the com- mon infections in the developing world. ${ }^{1}$ Conventional drug therapy for this infection is becoming increasingly ineffective with the emergence of multidrug resistant strains. ${ }^{3,10}$

In this study, the isolated strains of salmonella typhi were resistant to conventional drugs, both in vivo and in vitro. The nature of factors contributing to the occurence of the antimicrobial resistant salmonella typhi infection have not been evaluated by us, though widespread indiscriminate use of antimicrobials is one possible explanation and is reported in other non-typhoidal salmonella infections. ${ }^{13}$

There remains a need for agents effective against multiple drug resistant salmonella typhi infections. Since the isolated stains were resistant to all conventional drugs and since newer cephalosporins, reportedly effective against salmonella typhi strains, were not available, Norfloxacin was used. This drug has a remarkably broad antimicrobial spectrum. The use of the drug is also justified by in vitro studies, ${ }^{3,4}$ wherein Norfloxacin inhibited salmonella strains resistant to Ampicillin, Chloramphenical, and Contrimoxazole.

Chloramphenical resistant thyphoid fever is increasing. Its side effect of agranulocytosis cannot be sufficiently underscored, justifying a hunt for an effective and safe agent. Resistance to Norfloxacin is not easily produced ${ }^{3-7}$ and the drug has few side effects. ${ }^{12}$ We were able to obtain an excellent cure rate with no apparent adverse reactions. The tolerability of Norflaxin was excellent and compares with the study of use of Norfloxacin to treat chronic typhoid carriers. ${ }^{14}$

To our knowledge the use of Norfloxacin in the treatment of typhoid fever has not previously been reported in the literature. This study is the first of its kind, though the use of Ciprofloxacin in acute typhoid fever has been reported. ${ }^{13}$ Clinical efficacy of the drug in the present study demands further clinical trails to determine its efficacy in acute typhoid fever.

We conclude that Norfloxacin shows promise as an antibacterial agent for acute typhoid fever because of its excellent clinical response, minimal side effects and the elimination of the potential salmonella carrier state. It has proven efficacious in severe salmonella septecemia presenting with neuropsychiatric manifestations and also in multiple drug resistant strains.

\section{References}

1. Guerrant RL, Hook EW. Salmonella infections. Harrisons J of Int Med. 1983, 10th Edition: 957-65.

2. Norrby SR, Jonsson M. Antibacterial activity of Norfloxcin. Antimicrob Agents Chemother. 1983;23:15-8. 
3. Shungu DL, Weinberg E, Gadebusch $\mathrm{HH}$. In vitro antibacterial activity of Norfloxacin and other agents against gastrointestinal tract pathogens. Antimicrob Agents Chemother. $1983 ; 23: 86$.

4. Wolfson JS, Hooper DC. The fluroquinolones: Structures, mechanisms of action and resistance and spectra of activity in vitro. Antimicrob Agents Chemother. 1985;28:581-6.

5. Cruplin GC, Kenwright M, Hirst T. Investigations into mechanism of action of the antibacterial agent Norfloxacin. Antimicrob Agents Chemother. 1984 (Suppl B);12:9-23.

6. Hooper DC, Wolfson JS. The fluroquinolones pharmacology, clinical uses and toxicities in humans. Antimicrob Agents Chemother. 1985;28:700-21.

7. Seisser J, Weidemann, B. Elimination of plasmids by new 4-quinolones. Antimicrob Agents Chemother. 1985;28:700-2.

8. Panikar CKJ, Vimla KN. Transferable chloramphenicol resistance in salmonella typhi. Nature. 1972;239:109-13.

9. Neu HC, Cherubin CE, Longo ED, et al. Antimicrobial resistance and $\mathbf{R}$ factor transfer among isolates of salmonella in north eastern USA. A comparison of human isolates. J Infect Dis. 1975; 132:617-22.

10. Ryder RW, Black PA, Murlin AC, et al. Increase in antibiotic resistance among isolates of salmonella in US, 1967-1975. J Infect Dis. 1980;142:485-91.

11. Goyal A, Gupta MK, Sooch N. Salmonella Surveillance Bulletin, National Salmonella and Eschorichie Center, Kasauli, India, 1985;9:3.

12. Wolfson JS, Hooper DC. Norfloxacin: A new targeted fluroquinolone antimicrobial agent. Ann Int Med. 1988;108-238-51.

13. Spika JS, Waterman SH, Solex, GW, et al. Chloramphenical resistant salmonella-newport traced through hamburger and dairy forms: A major persisting source of salmonella in California. New Engl J Med. 1987;316:565.

14. Edurardo G, Jorge GG, Luis B, et al. Use of Norfloxacin to treat chronic typhoid carrier. J Infect Dis. 1988;157:1221-5.

15. Dupont HL, Ericsson CD, Robinson A, et al. Current problems in antimicrobial therapy for bacterial enteric infections. Am J Med. 1987;82(Suppl 3):323-8. 